Rabaska

Revue d'ethnologie de l'Amérique française

LAMARRE, JEAN. Les Canadiens français du Michigan. Leur contribution dans le développement de la vallée de la Saginaw et de la péninsule de Keweenaw 1840-1914. Québec, Septentrion, 2000, 224 p. ISBN 2-89448-146-2

\title{
Normand Beaupré
}

Volume 4, 2006

URI : https://id.erudit.org/iderudit/201787ar

DOI : https://doi.org/10.7202/201787ar

Aller au sommaire du numéro

Éditeur(s)

Société québécoise d'ethnologie

ISSN

1703-7433 (imprimé)

1916-7350 (numérique)

Découvrir la revue

Citer ce compte rendu

Beaupré, N. (2006). Compte rendu de [LAMARRE, JEAN. Les Canadiens français du Michigan. Leur contribution dans le développement de la vallée de la Saginaw et de la péninsule de Keweenaw 1840-1914. Québec, Septentrion, 2000, 224 p. ISBN 2-89448-146-2]. Rabaska, 4, 175-177. https://doi.org/10.7202/201787ar d'utilisation que vous pouvez consulter en ligne. 
LAMARRE, JEAN. Les Canadiens français du Michigan. Leur contribution dans le développement de la vallée de la Saginaw et de la péninsule de Keweenaw 1840-1914. Québec, Septentrion, 2000, 224 p. ISBN 2-89448146-2.

Il est bien nécessaire pour ceux qui s'intéressent aux diverses facettes de l'histoire et de la culture canadiennes-françaises qu'il y ait des ouvrages qui poursuivent les points dominants des différentes étapes historiques et socioéconomiques du peuple québécois. Jean Lamarre apporte, par ce livre sur les Canadiens français du Michigan, sa contribution consacrée à l'étude de l'évolution de ce peuple « aux semelles de vent ».

Premièrement, l'étude de Jean Lamarre s'avère l'ouvrage d'un chercheur fidèle à la recherche détaillée. Il nous parle non seulement de l'histoire, mais aussi des faits socio-économiques à propos de la mouvance des Canadiens français dès le $\mathrm{XVII}^{\mathrm{e}}$ siècle et jusqu'au début du XIX'e siècle. Il nous rappelle ce « vaste empire dont les limites s'étendaient jusqu'au Grand Nord, à l'ouest, au-delà du fleuve Mississippi, à l'est, jusqu'aux colonies anglaises, au sud, jusqu'au golfe du Mexique ". Afin de mieux nous situer, il note qu'avant les années 1860 les Canadiens français se sont dirigés vers la Nouvelle-Angleterre et le Midwest américain. Et les chiffres démontrent qu'en 1890 plus d'un demi-million d'individus d'origine canadienne-française vivaient aux ÉtatsUnis dont la grande majorité habitait les États du Nord-Est. Un peu plus de cinquante-huit mille habitaient le Michigan. Et puis l'auteur cherche à savoir comment ces immigrants se sont adaptés à la réalité industrielle au Michigan. C'est alors que l'ouvrage se concentre sur cet État.

Ce qui rend cette œuvre fascinante, c'est que l'auteur a eu souci d'élargir son étude au-delà d'un État particulier. Il inclut la Nouvelle-Angleterre et l'État de New-York ainsi que d'autres endroits où s'installèrent ces immigrants. D'abord, il y eut cette même grande cause de cette migration vers les États-Unis : la pauvreté surtout au Bas-Canada, telle qu'énoncée dans le rapport de Louis Perrault en 1849. Apparemment, c'était ou crever sur une « terre " ou « mouver ».

L'étude trace l'évolution du déplacement sur le continent en décrivant les divers attraits du développement économique, géographique et social pour un Canadien français qui tâche de survivre. En premier lieu, il y a la traite des fourrures. C'est elle qui est au cœur de ce développement, nous rappelle Lamarre. La traite des fourrures débute au Québec à partir du XVII ${ }^{e}$ siècle et se prolonge jusqu'au début du XIX ${ }^{\mathrm{e}}$ siècle. Puis, " les Grands Lacs ont constitué la plaque tournante autour de laquelle s'est articulé le commerce des fourrures " insiste l'auteur. Vient ensuite l'industrie forestière. L'insuffisance de l'agriculture entraîne le fermier vers les chantiers surtout de l'État du Maine, ce « Pine Tree State », et en petites concentrations vers 
l'État du Vermont. L'auteur signale aussi l'attrait des industries manufacturières du coton, de la laine et des usines de chaussures en Nouvelle-Angleterre. Quant au Midwest américain, il faut inclure l'Indiana, l''Illinois, le Michigan, le Minnesota, l'Ohio et le Wisconsin. Cependant, « l'État du Michigan s'est avéré la destination par excellence des Canadiens français tout au long du $\mathrm{XIX}^{\mathrm{e}}$ siècle ». Lamarre nous rappelle aussi que la prospection minière fut très importante pour les immigrants canadiens-français.

C'est après nous avoir parlé de l'état du Québec et de ses habitants vis-àvis de leur condition de survie que l'auteur se concentre ensuite sur le développement de la vallée de la Saginaw et de la péninsule de Keweenaw au Michigan. Il nous présente plusieurs exemples d'immigrants d'origine québécoise avec des chiffres à l'appui. Vers les années 1850 , le nombre de Canadiens français dans la vallée était tel qu'il nécessitait un regroupement sur une base linguistique et religieuse pour la « survivance » culturelle, affirme Lamarre. Fait intéressant, il découvre qu'un bon nombre de ces immigrants travaillaient comme propriétaires d'hôtels, propriétaires de " saloons 》 et commerçants d'alcool; et l'auteur s'en étonne puisque « les études passées ne nous ont pas habitué à associer aussi étroitement les Canadiens français au commerce de l'acool $»$. Vers la fin de ce chapitre, l'auteur propose la théorie que « les Canadiens français de la vallée ont participé à un processus migratoire qui pourrait être qualifié de triangulaire, liant, dans un mouvement giratoire, le Québec, le Nord-Est américain et la vallée de la Saginaw ». Très intéressante, cette observation.

La partie sur la péninsule de Keweenaw suit le même modèle que l'étude de la vallée de Saginaw avec ses exemples particuliers et ses chiffres. L'auteur nous informe que le premier attrait pour les immigrants de la péninsule fut le développement minier. Par la suite, il étudie le village de Calumet et sa vie communautaire, une communauté cosmopolite et multiethnique. Outre les quelques précisions particulières, ce chapitre me paraît moins intéressant.

Dans sa conclusion, Lamarre résume ce qu'il a déjà énoncé dans son étude. Ce que je trouve le plus intéressant, c'est la comparaison qu'il fait entre les caractéristiques de la migration vers le Michigan avec celles en Nouvelle-Angleterre, c'est-à-dire que le Canadien français de la NouvelleAngleterre s'est intégré au marché du travail manufacturier qui faisait appel surtout à une main-d'œuvre féminine et juvénile bon marché. Au Michigan, le Canadien français, très familier avec le marché du travail forestier, s'est vite adapté à cette zone « frontière » où le caractère socioculturel n'était pas encore complètement établi, « alors qu'en Nouvelle-Angleterre le Canadien français se retrouvait dans des centres urbains où la réalité sociale était bien établie ». 
Voilà une étude soignée et bien documentée sur le Michigan. Cependant le lecteur, souhaiterait un peu plus d'éléments humains, telles des histoires orales et des anecdotes qui viendraient revigorer une étude scientifique de cette nature.

Normand Beaupré

Professeur émérite

Université de la Nouvelle-Angleterre, Biddeford (Maine) 